\title{
Correlation between Autistic Traits and Gait Characteristics while Two Persons Walk Toward Each Other
}

\author{
Masahiro Shigeta,, ,\# Akira Sawatome,,${ }^{* *}$ Hiroko IchiKawa, ${ }^{* * *}$ Hiroshi TaKemura ${ }^{*}$
}

\begin{abstract}
Gait characteristics vary among people and correspond to individual differences of external and internal traits. Recent studies on autism spectrum disorders (ASD) reported that gait characteristics are associated with a walker's autistic trait. Previous studies measured gait characteristics with walking alone and did not investigate gait characteristics in interactive situations. The goal of this study was to examine the correlation between ASD traits and gait characteristics in typically developed (TD) young adults. The subjects completed a Subthreshold Autism Trait Questionnaire (SATQ) for quantitative measurement of autistic traits. After completing the questionnaire, the subjects participated in walking experiments in pairs using an inertial measurement unit (IMU)-type three-dimensional motion capture system. Two participants walked toward each other and had to avoid collision with their counterparts. The norm in the $\mathrm{X}$ - and $\mathrm{Y}$-axis directions of acceleration, and the norm in the X-, Y-, and Z-axis directions of the angular velocity of four body parts (waist, left/right foot, and head) were calculated. In this study, the standard deviation of each norm and the average pitch were used as evaluation indices of the magnitude of sway of the body. Each parameter was calculated in two areas: the Walking Area (from the 3rd to 6th steps) and the Passing Area (from the 7th to 10th steps). Multiple regression analysis was performed to determine the factors that explain the SATQ scores. All explanatory variables were standardized, and a multiple linear regression analysis was performed. The results revealed that when the distance between the two subjects at the time of passing each other was $20 \mathrm{~cm}$, there was a strong correlation between the SATQ score and the standard deviation of the norm of the angular velocity of the waist from the 7th to 10th steps. This finding suggests that compared with TD individuals, individuals with severe ASD traits significantly increase the standard deviation of the norm of the angular velocity of the waist in order to avoid contact with their counterparts.
\end{abstract}

Keywords: ASD, motion capture system, accelerometer, exhaustive search, collision avoidance.

Adv Biomed Eng. 7: pp. 55-62, 2018.

\section{Introduction}

Gait characteristics vary among people and correspond to individual differences of external and internal traits. Generally, a tall person walks with a longer stride than a short person, and an elderly person walks more slowly than a young person. Many psychological studies on biological motion [1] have demonstrated that we can rec-

This study was presented at the Symposium on Biomedical Engineering 2017, Ueda, September, 2017.

Received on July 21, 2017; revised on October 30, 2017; accepted on December 8, 2017.

* Department of Mechanical Engineering, Faculty of Science and Technology, Tokyo University of Science, Chiba, Japan.

** Japan Society for the Promotion of Science, Tokyo, Japan.

**** Liberal Arts, Faculty of Science and Technology, Tokyo University of Science, Chiba, Japan.

\# 2641 Yamazaki, Noda, Chiba 278-8510, Japan.

E-mail: 7517627@ed.tus.ac.jp ognize a walker's internal state, such as the gender [2] and emotional state [3], by observing the motion of several point light markers attached to the joints of the walker. Troie [4] reviewed previous articles and suggested that even personal traits can be recognized by observing gait characteristics.

Recent studies on autism spectrum disorders (ASDs) have reported that gait characteristics are associated with a walker's autistic traits. ASD is a neurodevelopmental disorder characterized by persistent deficits in social communication and social interaction, and restricted, repetitive patterns of behavior, interests, or activities [5]. Although motor coordination deficits are not defined as fundamental aspects of ASD, a meta-analysis of the relationship between ASD and motor control impairments concluded that individuals with ASD have atypical features in gait and balance control [6].

An increasing number of researchers have reported that individuals diagnosed with ASD show gait move- 
ments different from typically developed (TD) control participants [7, 8]. Vilensky et al. [9] found significant atypical characteristics in the gait of individuals with ASD and those who had profound disorders of communication and social relatedness. Previous studies [6] measured gait characteristics of an individual walking alone and did not investigate gait characteristics in interactive situations.

ASD traits, which include social communication impairment, should correlate with gait characteristics as two individuals are walking toward each other. Honma et al. [10] measured the gait characteristics of two individuals walking toward each other. In this situation, the walking pattern of each individual is interrupted by the counterpart. Then, the walker must move aside from the preplanned trajectory to avoid a collision. Each walker has to observe the counterpart's movement carefully and predict the direction of the counterpart's movement, while individuals with severe ASD traits are not good at predicting another person's intentions.

The gait characteristics measured using an accelerometer attached to the body parts of a human walker are expected to describe the characteristics of individuals with ASD. The spatial and temporal characteristics measured using a walkway, such as stride length and duration variation, have been reported to differ in children with ASD compared with those in typically developing children [11]. Naruse et al. [12] used an optical three-dimensional motion capture system and investigated the gait characteristics of children with attention-deficit hyperactivity disorder (ADHD), which is often a comorbid disorder linked with ASD [13]. They found that an increased anterior pelvic angle was associated with ADHD symptoms.

The goal of this study was to examine the correlation between ASD traits and gait characteristics in TD individuals. ASD traits are currently known to manifest a broad range of conditions. ASD is a comorbid disorder that affects not only individuals diagnosed with ASD but also a certain population of TD people who also suffer from ASD symptoms.

In this study, we measured the gait characteristics of TD individuals using an inertial measurement unit (IMU)-type three-dimensional motion capture system to determine which gait characteristic parameters correlate with ASD traits. Two participants walked toward each other and had to avoid collision with their counterparts. IMU sensors were attached to 16 body parts of the walker. The severity of ASD traits was assessed by a Subthreshold Autism Trait Questionnaire (SATQ) [14], which is a questionnaire for broad autism conditions among TD individuals.

\section{Methods}

Fourteen pairs of healthy young adult males participated in the study. The mean (standard deviation) height, age, and SATQ score of the subjects were $171.76 \mathrm{~cm}$ (S.D. 5.49), 21.68 years (S.D. 1.22), and 36.00 (S.D. 11.35), respectively. The dominant foot was the right in 26 subjects, while two subjects did not answer. This study was conducted in accordance with the ethical principles of the Helsinki Declaration, and after obtaining informed consent from each subject. The study was approved by the Ethics Committee of Tokyo University of Science (approval number 16027). The test procedures and possible risks were explained to each subject prior to obtaining written informed consent.

The subjects completed the SATQ, a questionnaire consisting of 24-item self-report scales for measuring quantitative autistic traits. For each scale, the subjects rated themselves on a four-point Likert scale ranging from "false, not at all" (0) to "very true" (3). The total SATQ score was calculated as the sum of the 24-item scores. A high SATQ score indicates increased severity of autistic traits. Figure 1 shows the distribution of the SATQ scores.

After completing the SATQ, the subjects participated in walking experiments in pairs after putting on the IMU-type three-dimensional motion capture system (NOITOM Ltd. Perception Neuron). Two subjects stood facing each other, and were instructed to pass each other and avoid a collision. Figure 2 shows a schematic diagram of the walking experiment. Two subjects started walking with the same step length (fixed toe positions) at the same time while hearing a timing sound. From the 1st to the 6th step (Walking Area), they synchronized the time of heel contact to the sound of a 120-bpm metronome. After the 6th step (Passing Area), the metronome was turned off, and the participants were allowed to walk

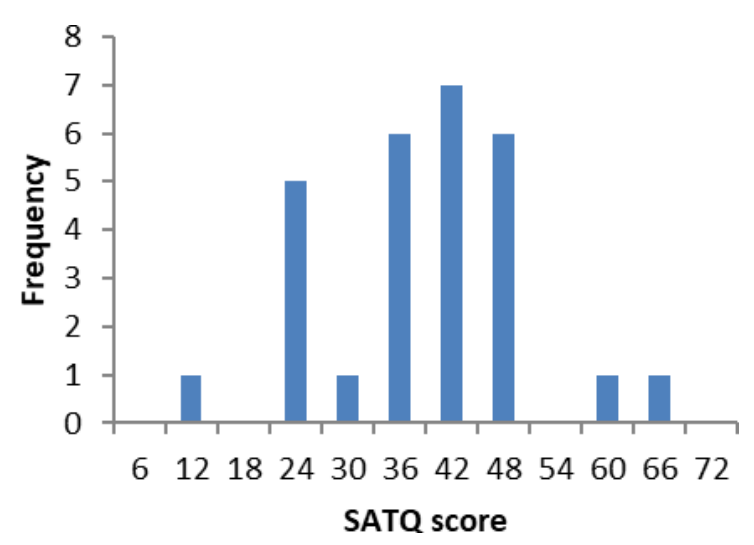

Fig. 1 Distribution of the total SATQ scores for all subjects. 


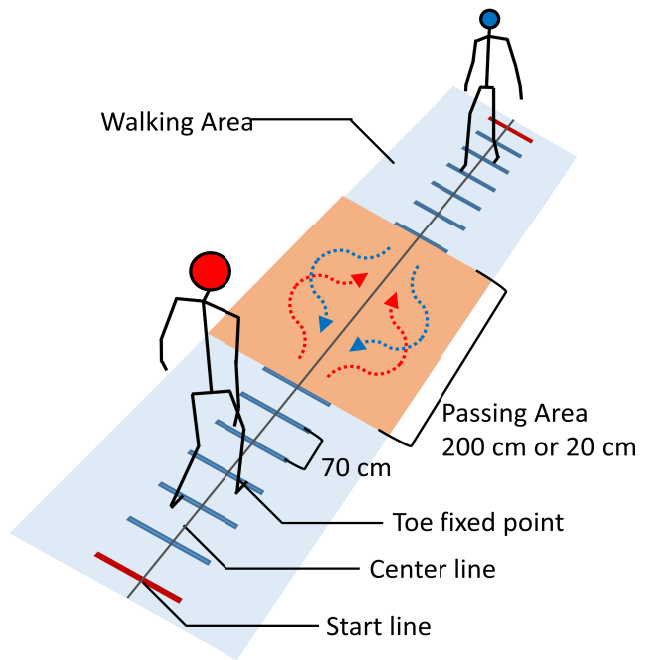

(a) Schematic diagram

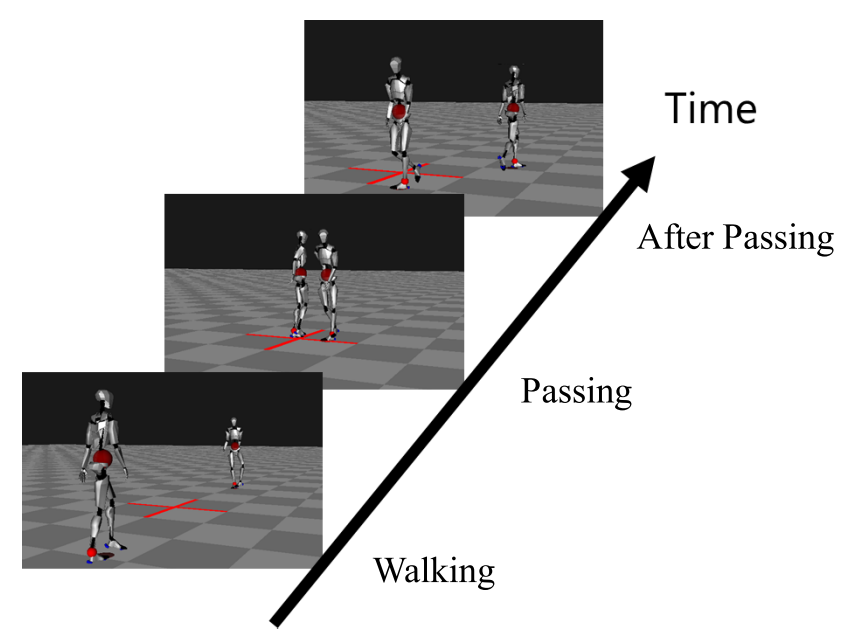

(b) Flow of experiment

Fig. 2 Overview of walking experiment.

freely, avoiding their counterparts. They returned to the center line again and walked through their counterparts' walking areas, ignoring the fixed-toe positions.

There were four experimental conditions with two variants: (1) knowing the direction to avoid each other beforehand (known condition) or not (unknown condition), and (2) length of the Passing Area being $20 \mathrm{~cm}$ or $200 \mathrm{~cm}$. Under the known condition, the subjects were notified of the avoidance direction before each trial. The order of avoidance direction and pair conditions were counterbalanced across participants. For each experimental condition, the subjects repeated six trials. All subjects completed 24 trials in total.

The sensor data was corrected using Axis Neuron software (NOTIOM Ltd.). The corrected data was calculated using the global coordinate system. The subject's motion was recorded at $120 \mathrm{fps}$. The contact data calculated using the acceleration and angular velocity were used for analysis. Any plainly incorrect contact data was adjusted manually. A fourth-order high-pass Butterworth filter with a cutoff frequency of $0.001 \mathrm{~Hz}$ and a fourth-order low-pass Butterworth filter with a cutoff frequency of $5 \mathrm{~Hz}$ were applied to the extracted data.

The norm in the $\mathrm{X}$ - and $\mathrm{Y}$-axis directions of acceleration, and the norm in the $\mathrm{X}$-, $\mathrm{Y}$-, and $\mathrm{Z}$-axis directions of the angular velocity of four body parts (waist, left/right foot, and head) were calculated. IMU sensors are often used to measure the motion of these body parts. Furthermore, the pitch can be measured from the motion of the foot [15]. In this study, the standard deviation of each norm and the average pitch were used as evaluation indices of the degree of sway of the body. Each parameter was calculated in two areas: Walking Area (from 3rd to 6th steps) and Passing Area (from 7th to 10th steps). The total number of parameters were 18 (pitch $\times 2$ areas, S.D. of norm of acceleration $\times 4$ parts $\times 2$ areas, and S.D. of norm of angular velocity $\times 4$ parts $\times 2$ areas).

Equations (1), (2), (3), and (4) indicate the norm of acceleration $\left(a_{N}\right)$ and its standard deviation $\left(a_{S . D .}\right)$, and the norm of the angular velocity $\left(\omega_{N}\right)$ and its standard deviation $\left(\omega_{S . D .}\right)$. A larger standard deviation indicates that the person makes a large movement and bending motion. The average and S.D. were calculated for each trial. The raw data for the waist are shown in Fig. 3. The upper six graphs show the acceleration and angular velocity in the $\mathrm{X}-$, Y-, and Z-axis directions, and the lower two graphs show the acceleration and angular velocity norms. The dotted lines indicate a typical subject with a low SATQ score, and the solid lines indicate a typical subject with high SATQ score. The mean values for each of the six trials were then calculated.

$$
\begin{aligned}
& a_{N}=\sqrt{a_{x}^{2}+a_{y}^{2}} \\
& a_{S . D .}=\sqrt{\frac{1}{n} \sum_{i=1}^{n}\left(a_{N i}-\overline{a_{N}}\right)^{2}} \\
& \omega_{N}=\sqrt{\omega_{x}^{2}+\omega_{y}^{2}+\omega_{z}^{2}} \\
& \omega_{S . D .}=\sqrt{\frac{1}{n} \sum_{i=1}^{n}\left(\omega_{N i}-\overline{\omega_{N}}\right)^{2}}
\end{aligned}
$$

Multiple regression analysis was performed to determine the factors that explain the SATQ score. In order to choose effective explanatory variables, we conducted the multiple regression analysis using two variable selection methods. One was the well known stepwise method using a conventional algorithm. The other was an exhaustive search method [16-19] in which multiple regression 

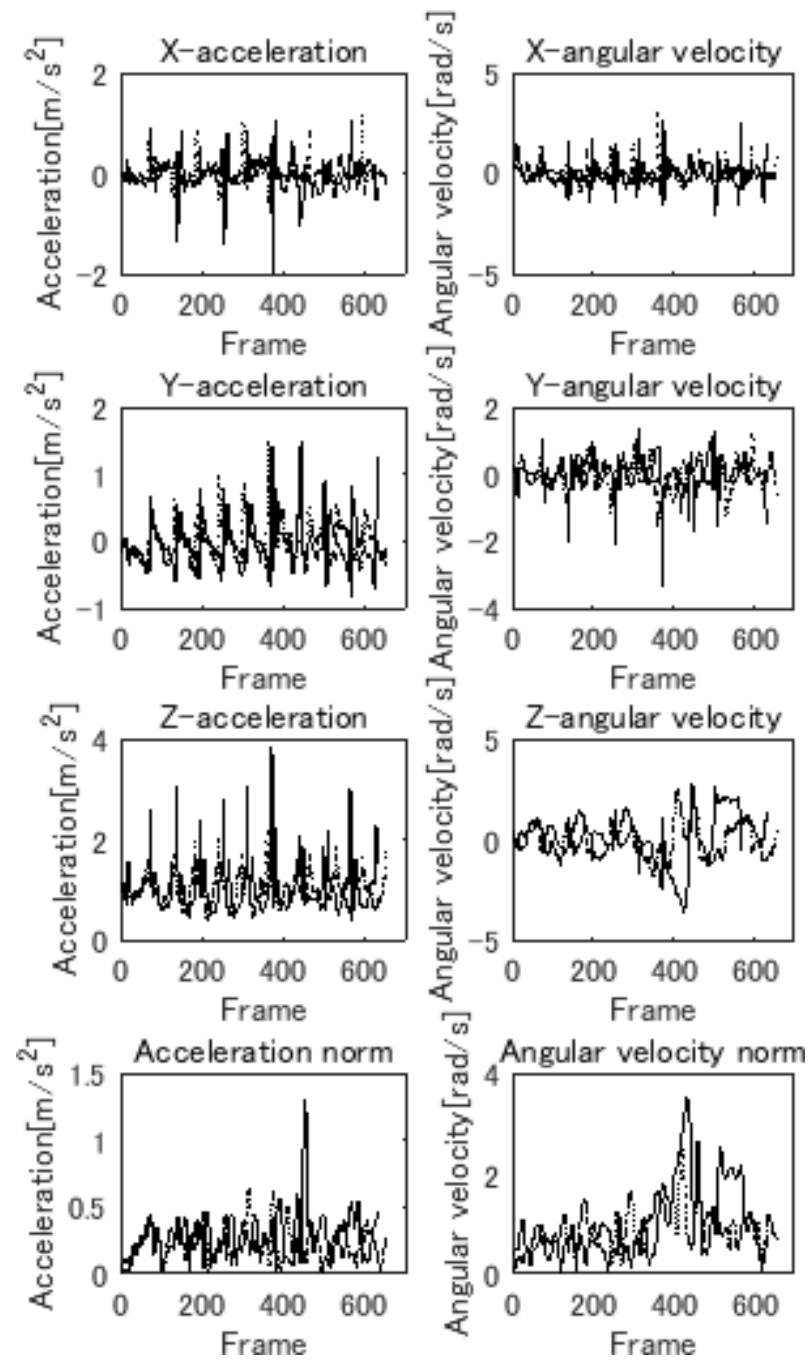

Fig. 3 Raw data for the waist. The dotted lines indicate a subject with a low SATQ score, and the solid lines indicate a subject with high SATQ score. analysis was performed on each of all $2^{9}-1$ combinations of the nine variables in each Walking Area and Passing Area.

The results of the multiple regression analysis were evaluated using the cross-validation error (CVE). The CVE is the root mean square error (RMSE) of the difference between the SATQ score predicted by cross validation and the actual SATQ score. Specifically, cross validation was performed using leave-one-out cross validation (LOOCV). The SATQ score predicted that multiple regression analysis was performed using the data for 27 out of 28 participants and that the value of the explanatory variable of the remaining participant was substituted.

When the SATQ scores of all the subjects were similarly predicted, 28 types of error were calculated, and the RMSE was calculated from each error. This was defined as the CVE. All statistical analyses were conducted using MATLAB version 2017a (MathWorks, Inc).

\section{Results}

First, under each condition, we compared the average values of each of the 18 variables between the Walking Area and the Passing Area. There were significant differences in all parameters excluding $a_{S . D}$. and $\omega_{S . D}$. of the right foot. Therefore, the analysis was performed for the Walking Area and Passing Area separately.

Table 1 presents the correlation coefficients between the 18 factors and SATQ scores for each condition. As a result of performing the test for non-correlation by adjusting the significance level using the Bonferroni method, there was a significant correlation between the SATQ score and the $a_{S . D}$. or $\omega_{S . D}$. of the waist for the Passing Area in the $20-\mathrm{cm}$ condition. In the

Table 1 Pearson's correlation coefficients between SATQ score and walking parameters. $\left({ }^{* *} p<.0007\right.$, ${ }^{*} p<.05$; corrected by Bonferroni method).

\begin{tabular}{|c|c|c|c|c|c|c|c|c|c|}
\hline & & \multicolumn{4}{|c|}{ Walking } & \multicolumn{4}{|c|}{ Passing } \\
\hline & & \multicolumn{2}{|c|}{$20 \mathrm{~cm}$} & \multicolumn{2}{|c|}{$200 \mathrm{~cm}$} & \multicolumn{2}{|c|}{$20 \mathrm{~cm}$} & \multicolumn{2}{|c|}{$200 \mathrm{~cm}$} \\
\hline & & Known & Unknown & Known & Unknown & Known & Unknown & Known & Unknown \\
\hline Pitch & & 0.13 & -0.05 & 0.14 & -0.01 & -0.16 & -0.13 & 0.19 & 0.02 \\
\hline \multirow[t]{2}{*}{ Waist } & $a_{S . D .}$ & -0.22 & -0.16 & -0.24 & -0.23 & ${ }^{*} 0.43$ & ${ }^{*} 0.42$ & 0.08 & 0.23 \\
\hline & $\omega_{S . D}$ & 0.13 & 0.10 & 0.10 & 0.14 & ${ }^{*} 0.58$ & ${ }^{* *} 0.64$ & 0.36 & 0.25 \\
\hline \multirow[t]{2}{*}{ Right foot } & $a_{S . D .}$ & -0.04 & -0.07 & -0.18 & -0.15 & 0.17 & 0.36 & 0.11 & 0.05 \\
\hline & $\omega_{S . D}$ & -0.07 & -0.07 & -0.24 & -0.23 & 0.36 & ${ }^{*} 0.51$ & 0.14 & 0.15 \\
\hline \multirow[t]{2}{*}{ Left foot } & $a_{S . D .}$ & -0.35 & -0.26 & -0.29 & -0.30 & -0.02 & 0.14 & -0.13 & -0.16 \\
\hline & $\omega_{S . D}$ & 0.03 & 0.03 & 0.02 & 0.05 & 0.06 & 0.25 & 0.20 & 0.24 \\
\hline \multirow[t]{2}{*}{ Head } & $a_{S . D .}$ & -0.08 & -0.17 & -0.13 & -0.11 & 0.30 & 0.28 & 0.18 & 0.16 \\
\hline & $\omega_{S . D}$ & 0.14 & 0.09 & 0.12 & 0.01 & 0.17 & 0.28 & 0.12 & 0.16 \\
\hline
\end{tabular}


20-cm and unknown condition, there was a significant correlation between the SATQ score and $\omega_{S . D}$. of the right foot for the Passing Area. The trends for the known and unknown conditions were similar.

Next, multiple regression analysis was performed to determine the factors that explain the SATQ score. We conducted multiple regression analyses using nine standardized numerical values as the explanatory variables and the SATQ score as the objective variable under each condition for the Walking Area and for the Passing Area. Table 2 presents the regression coefficients for the variables selected by the stepwise method of the multiple regression analysis. Under the $20-\mathrm{cm}$ and known conditions, $\omega_{S . D}$. of the waist was not selected as an explanatory variable for the Walking Area, but was selected for the Passing Area. The CVE was 9.89.

For the $20-\mathrm{cm}$ and unknown conditions, $\omega_{S . D}$. of the waist was selected as the explanatory variable only for the Passing Area. The result was the same as in the 20$\mathrm{cm}$ and known conditions. The CVE was 9.18. Table 3 presents the multiple regression analysis for the Passing Area for the 20-cm and known conditions, and the 20-cm and unknown conditions.

The same analysis was performed for the $200-\mathrm{cm}$ conditions, but no explanatory variables were selected for either the Walking Area or the Passing Area.

Based on the results of the $20-\mathrm{cm}$, known, and un- known conditions, we merged the results of the known and unknown conditions for further analysis. The data was merged by calculating the mean of 12 trials of the known and unknown conditions together, comprising 18 parameters calculated by the mean of six trials.

As a result of multiple regression analysis using the stepwise method for the $20-\mathrm{cm}$ and $200-\mathrm{cm}$ conditions, $\omega_{S . D}$ of the waist was selected as the explanatory variable only in the Passing Area for the 20-cm condition. The CVE was 9.28. The multiple regression analysis results are presented in Table 4. B is the coefficient; Std. Error $\mathrm{B}$ is the standard error of the coefficient; and $\beta$ is the standardizing coefficient. The horizontal axis of Figure 4 shows the actual SATQ scores, and the vertical axis shows the scores predicted by cross validation. The straight line is a graph of $\mathrm{y}=\mathrm{x}$. Explanatory variables were not selected for the $200-\mathrm{cm}$ condition.

To determine the best combination in the Passing Area in the $20-\mathrm{cm}$ condition, multiple regression analysis was performed using the exhaustive search method. Figure 5 shows the results. The horizontal axis indicates the rank-ordered selected feature subset. The vertical axis of the top panel is the CVE. The vertical axis of the bottom panel is the index of the explanatory variable. The regression equation with the smallest CVE is an equation using seven variables including the $\omega_{S . D}$. of the waist; the CVE was 9.03. Table 5 presents the results of

Table 2 Regression coefficients of multiple regression analysis using stepwise method.

\begin{tabular}{|c|c|c|c|c|c|c|c|c|c|}
\hline & & \multicolumn{4}{|c|}{ Walking } & \multicolumn{4}{|c|}{ Passing } \\
\hline & & \multicolumn{2}{|c|}{$20 \mathrm{~cm}$} & \multicolumn{2}{|c|}{$200 \mathrm{~cm}$} & \multicolumn{2}{|c|}{$20 \mathrm{~cm}$} & \multicolumn{2}{|c|}{$200 \mathrm{~cm}$} \\
\hline & & Known & Unknown & Known & Unknown & Known & Unknown & Known & Unknown \\
\hline Pitch & & - & - & - & - & - & - & - & - \\
\hline \multirow[t]{2}{*}{ Waist } & $a_{S . D .}$ & - & - & - & - & - & - & - & - \\
\hline & $\omega_{S . D}$ & - & - & - & - & 6.55 & 7.24 & - & - \\
\hline \multirow[t]{2}{*}{ Right foot } & $a_{S . D .}$ & - & - & - & - & - & - & - & - \\
\hline & $\omega_{S . D}$ & - & - & - & - & - & - & - & - \\
\hline \multirow[t]{2}{*}{ Left foot } & $a_{S . D .}$ & - & - & - & - & - & - & - & - \\
\hline & $\omega_{S . D}$ & - & - & - & - & - & - & - & - \\
\hline \multirow[t]{2}{*}{ Head } & $a_{S . D .}$ & - & - & - & - & - & - & - & - \\
\hline & $\omega_{S . D}$ & - & - & - & - & - & - & - & - \\
\hline
\end{tabular}

Table 3 Multiple regression analysis table $\left({ }^{*} p<.05,{ }^{* *} p<.01,{ }^{* * *} p<.001\right)$ B: Coefficient, Std. Error B: standard error of the coefficient, $\beta$ : standardized coefficient.

\begin{tabular}{ccrrrrr}
\hline & \multicolumn{3}{c}{ Passing_Known_20 cm } & \multicolumn{3}{c}{ Passing_Unknown_20 cm } \\
\cline { 2 - 7 } & B & Std. Error B & $\beta$ & B & Std. Error B & $\beta$ \\
\hline Waist Passing $\omega_{S . D}$ & 41.8 & 11.6 & $.577^{* *}$ & 46.6 & 11.1 & $.638^{* * * *}$ \\
\hline $\mathrm{R}^{2}$ & $.332^{* *}$ & & & $.384^{* * *}$ & & \\
\hline
\end{tabular}


Table 4 Multiple regression analysis table after merging Known and Unknown conditions. $\left({ }^{* * * *} p<.001\right)$ B: Coefficient, Std. Error B: Standard error of the coefficient, $\beta$ : Standardized coefficient.

\begin{tabular}{cccc}
\hline & \multicolumn{3}{c}{ Passing_20 cm } \\
\cline { 2 - 4 } & $\mathrm{B}$ & Std. Error B & $\beta$ \\
\hline Waist $\omega_{\text {S.D }}$ & 47.7 & 11.5 & $.632^{* * *}$ \\
\hline $\mathrm{R}^{2}$ & $0.38^{* * *}$ & & \\
\hline
\end{tabular}

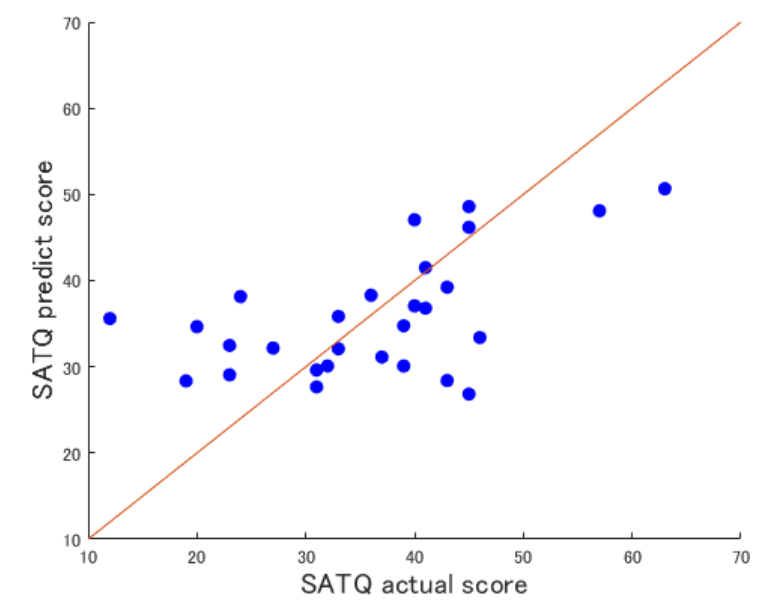

Fig. 4 Actual versus predict scores using stepwise method.
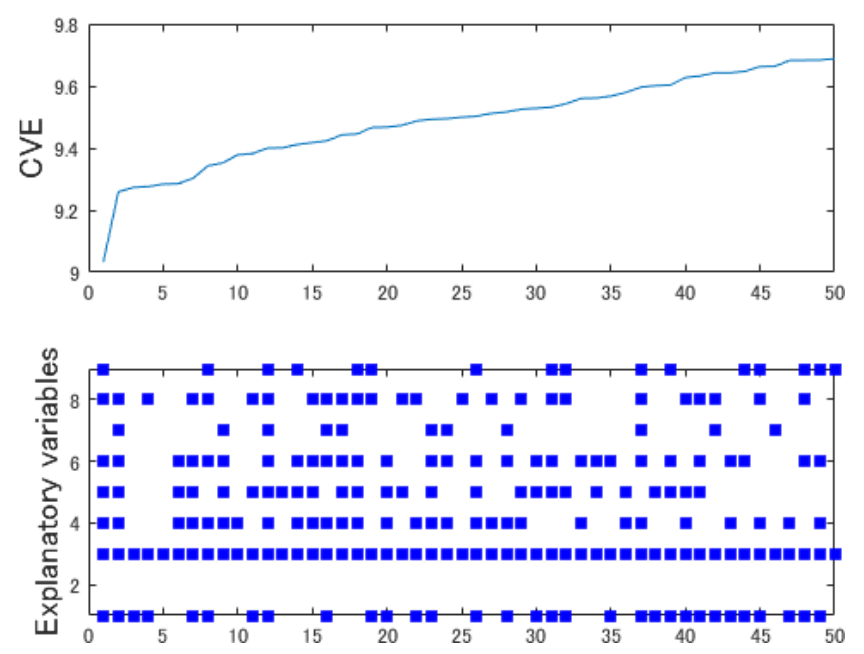

Fig. 5 CVE (top panel) and explanatory variables (bottom panel) corresponding to a regression model indexed by horizontal axis. Each number indicates 1: pitch, 2: Waist $a_{S . D .}, 3$ : Waist $\omega_{S . D .}, 4$ : Right foot $a_{S . D .}, 5$ : Right foot $\omega_{\text {S.D. }}, 6$ : Left foot $a_{S . D .}$, 7: Left foot $\omega_{\text {S.D. }}$, 8: Head $a_{\text {S.D. }}, 9:$ Head $\omega_{\text {S.D. }}$

multiple regression analysis when the CVE is smallest. Figure 6 shows the scatter plot and a straight line for $\mathrm{y}=$ $\mathrm{x}$.
Table 5 Multiple regression analysis table using exhaustive search. $\left({ }^{*} p<.05,{ }^{* *} p<.01,{ }^{* * *} p<.001\right) \mathrm{B}$ : Coefficient, Std. Error B: Standard error of the coefficient, $\beta$ : Standardized coefficient.

\begin{tabular}{crrr}
\hline & \multicolumn{3}{c}{ ES, Passing, 20 cm } \\
\cline { 2 - 4 } & B & Std. Error B & \multicolumn{1}{c}{$\beta$} \\
\hline Pitch & -.734 & .498 & -.247 \\
Waist $\omega_{S . D}$ & 46.6 & 14.3 & $.618^{* *}$ \\
Right foot $a_{S . D}$ & 75.9 & 43.6 & .367 \\
Right foot $\omega_{S . D}$ & 21.6 & 13.8 & .299 \\
Left foot $a_{S . D}$ & -103 & 46.1 & $-.544^{*}$ \\
Head $a_{S . D}$ & -21.6 & 15.9 & -.277 \\
Head $\omega_{S . D}$ & 8.39 & 6.61 & .249 \\
\hline $\mathrm{R}^{2}$ & $0.48^{* * * *}$ & & \\
\hline
\end{tabular}

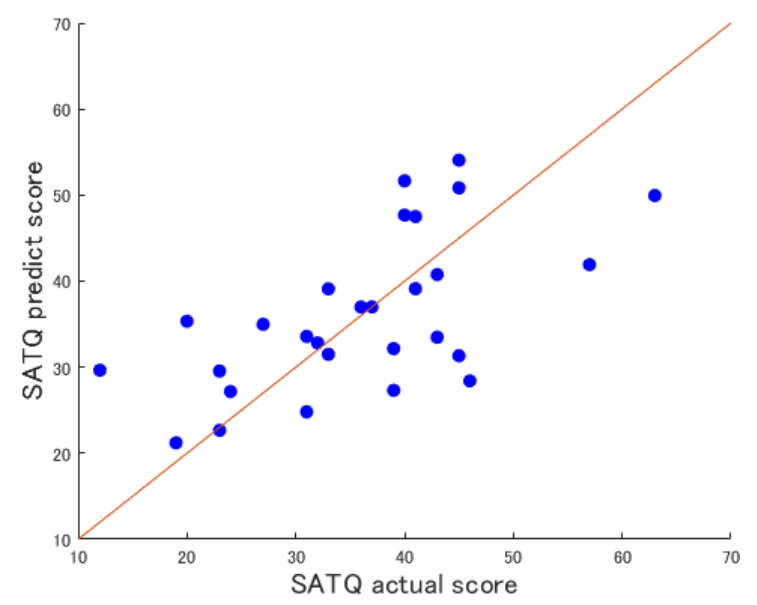

Fig. 6 Actual versus predict scores using exhaustive search.

\section{Discussion}

There were significant differences in many walking parameters between the Walking Area and the Passing Area. Correlation between the SATQ score and walking parameters was observed in the Passing Area and not in the Walking Area. By multiple regression analysis, we found that $\omega_{S . D}$. of the waist in the Passing Area for the 20-cm condition predicted the SATQ score.

Previous studies measured gait characteristics when a participant walked alone, but this study measured gait characteristics in an interactive situation when two participants walked toward each other. We found that $\omega_{S . D}$. of the waist, which reflects the magnitude of the waist movement, explained the severity of ASD traits in the interactive situation. Rinehart et al. [20] reported that the stride differed between individuals with ASD and TD persons, but the difference was not observed in this study 
because this study presented foot markers to the participants to maintain a fixed stride.

There was a significant correlation between the SATQ score and $\omega_{S . D}$. of the waist in the Passing Area under the $20-\mathrm{cm}$ and unknown conditions (Table 1). In addition, from the multiple regression analysis using the stepwise method, only $\omega_{S . D}$. of the waist in the Passing Area was selected as an explanatory factor under the 20$\mathrm{cm}$ and unknown conditions. These findings indicate that there is a strong relationship between participants with severe ASD traits and $\omega_{S . D}$. of the waist.

The role of the waist is to swing and bend. The closer the distance when passing the counterpart, the more it is necessary for a participant to twist the waist in order to avoid a collision. Recent studies reported that individuals with ASD maintained smaller personal spaces and tended to spatially approach each other at a closer distance than TD individuals [21,22]. Our findings suggested that individuals with more severe ASD traits showed a larger $\omega_{S . D}$. of the waist when passing the partner because they approached the partner more closely and started avoiding a collision later than TD individuals did.

From the multiple regression analysis using the stepwise method after merging the known and unknown conditions, only $\omega_{S . D}$. of the waist was selected as an explanatory factor under the $20-\mathrm{cm}$ condition, which was the same as that before merging. Furthermore, as a result of the exhaustive search, when examining the regression equation where the CVE was lowest, it was shown that $\omega_{S . D}$. of the waist was included. Table 5 also indicates that $\omega_{S . D}$. of the waist is a particularly important factor. These findings also show that there is a correlation between individuals with severe ASD traits and $\omega_{S . D}$. of the waist. We found significant correlation at $200 \mathrm{~cm}$ but no significant correlation at $200 \mathrm{~cm}$, probably reflecting that it is stressful for individuals with severe ASD traits to avoid contact with their partners at close distances.

\section{Conclusion}

The goal of this study was to investigate the correlation between gait characteristics and ASD traits in young adults. SATQ was used to measure ASD traits, and the behavior of avoiding collision between two subjects was used in the survey of gait characteristics. The results indicate that there is a significant correlation between ASD traits and the angular velocity of the waist. A previous study found a difference in stride length between individuals with ASD and TD persons while walking alone. However, the same finding was not observed in the present study, because we maintained a fixed stride in all subejcts. The present results suggest that gait characteristics in interpersonal situations reflect the severity of ASD traits. This study also illustrates that identification of walking parameters that explain ASD traits can be conducted easily using IMU sensors.

\section{Acknowledgement}

This research was supported by the Research Fund of Tokyo University of Science for Brain Interdisciplinary Research Division (BIRD) and a Grant-in-Aid for Scientific Research on Innovative Areas, "Sparse Modeling" from JSPS KAKENHI (16H01555 to HI).

\section{References}

1. Johansson G: Visual perception of biological motion and a model for its analysis. Percept Psychophys. 14, pp. 201-211, 1973.

2. Kozlowski LT, Cutting J: Recognizing the sex of a walker from a dynamic point-light display. Percept Psychophys. 21, pp. 575580, 1977.

3. Pollick FE, Sanford A: Perceiving affect from arm movement. Cognition. 82, pp. B51-B61, 2001.

4. Troje NF: Decomposing biological motion: A framework for analysis and synthesis of human gait patterns. J Vis. 2, pp. 371387, 2002.

5. American Psychiatric Association: Diagnostic and Statistical Manual of Mental Disorders, Fifth Edition. 2013.

6. Fournier KA, Hass CJ, Naik SK, Lodha N, Cauraugh JH: Motor coordination in autism spectrum disorders: A synthesis and meta-analysis. J Autism Dev Disord. 40, pp. 1227-1240, 2010.

7. Dufek JS, Eggleston JD, Harry JR, Hickman RA: A comparative evaluation of gait between children with autism and typically developing matched controls. Med Sci. 5, pp. 1-11, 2017.

8. Eggleston D, Harry JR, Hickman RA, Dufek JS: Analysis of gait symmetry during over-ground walking in children with autism spectrum disorder. Gait Posture. 55, pp. 162-166, 2017.

9. Vilensky JA, Damasio AR, Maurer RG: Gait dis- turbances in patients with autistic behavior: A preliminary study. Arch Neurol. 38, pp. 646-649, 1981.

10. Honma M, Koyama S, Kawamura M: Hesitant avoidance while walking: An error of social behavior generated by mutual interaction. Front Psychol. 6(1013), pp. 1-8, 2015.

11. Weiss MJ, Moran MF, Parker ME, Foley JT: Gait analysis of teenagers and young adults diagnosed with autism and severe verbal communication disorders. Front Integr Neurosci. 7(33), pp. 1-10, 2013.

12. Naruse H, Fujisawa T, Yatsuga C, Kubota M, Matsuo H, Takiguchi S, Shimada S, Imai Y, Hiratani M, Kosaka H, Tomoda A: Increased anterior pelvic angle characterizes the gait of children with attention deficit/hyperactivity disorder (ADHD). PLoS One. 12, e0170096, pp. 1-13, 2017.

13. Hofvander B, Delorme R, Chaste P, Nydén A, Wentz E, Ståhlberg O, Herbrecht E, Stopin A, Anckarsäter H, Gillberg C, Råstam M, Leboyer M: Psychiatric and psychosocial problem in adults with normal intelligence autismspectrum disorders. BMC Psychiatry. 9(35), pp. 1-9, 2009.

14. Nishiyama T, Suzuki M, Adachi K, Sumi S: Comprehensive comparison of self-administered questionnaires for measuring quantitative autistic traits in adults. J Autism Dev Disord. 44(5), pp. 993-1007, 2014.

15. Muller P, Steel T, Schauer T: Experimental evaluation of a novel 
inertial sensor based realtime gait phase detection algorithm. TAR 2015. 2015.

16. Garside MJ: The best sub-set in multiple regression analysis. J R Stat Soc Ser C. 14, pp. 196-200, 1965.

17. Ichikawa H, Kitazono J, Nagata K, Manda A, Shimamura K, Sakuta R, Okada M, Yamaguchi MK, Kanazawa S, Kakigi R: Novel method to classify hemodynamic response obtained using multi-channel fNIRS measurements into two groups: Exploring the combinations of channels. Front Hum Neurosci. 8(480), pp. 1-10, 2014.

18. Nagata K, Kitazono J, Nakajima S, Eifuku S, Tamura R, Okada M: An exhaustive search and stability of sparse estimation for feature selection problem. IPSJ Online Trans. 8, pp. 25-32, 2014.

19. Igarashi Y, Ichikawa H, Nakanishi-Ohno Y, Takenaka H, Kawabata D, Eifuku S, Tamura R, Nagata K, Okada M: A general framework for sparse variable selection: Exhaustive search with density-of-states estimation. J Phys Conf Ser. accepted.

20. Rinehart NJ, Tonge BJ, Bradshaw JL, Enticott PG: Gait function in high-functioning autism and Asperger's disorder. Eur Child Adolesc Psychiatry. pp. 256-264, 2006.

21. Kennedy DP, Adolphs R: Violations of personal space by individuals with autism spectrum disorder. PLoS One. 9(e103369), pp. 1-10, 2014.

22. Asada K, Tojo Y, Osanai H, Saito A, Hasegawa T, Kumagaya S: Reduced personal space in individuals with autism spectrum disorder. PLoS One. 11(e0146306), pp. 1-11, 2016.

\section{Masahiro SHIGETA}

Student, Graduate School of Science and Technology, Tokyo University of Science. Membership in Academic Societies: The Japan Society of Mechanical Engineers (JSME).

\begin{abstract}
Akira SAWATOME
Student, Graduate School of Science and Technology, Tokyo University of Science. 2017-Research Fellowship for Young Scientists, Japan Society for the Promotion of Science. Membership in The Japan Society of Mechanical Engineers (JSME) and Society of Biomechanics Japan (SOBIM).
\end{abstract}

\section{Hiroko ICHIKAWA}

Lecturer, Liberal Arts, Tokyo University of Science. She received the $\mathrm{PhD}$ degree in behavioral science from the University of Tsukuba, Japan, in 2005. She was Research Fellow of the Japan Society for the Promotion of Science and Assistant Professor at Chuo University. She is a member of The Japanese Psychological Association, The Japanese Psychonomic Association, and The Japanese Society of Child Neurology.
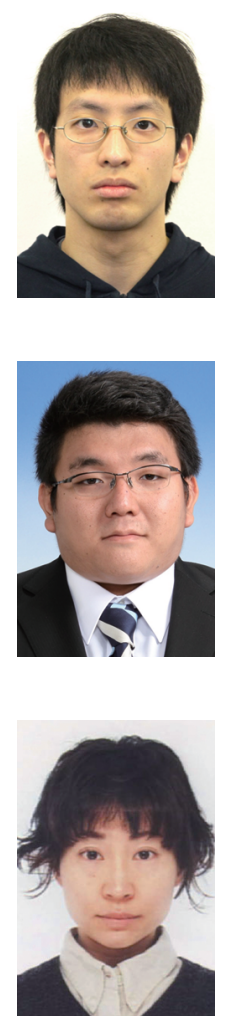
gy.

\section{Hiroshi Takemura}

Junior Associate Professor, Department of Mechanical Engineering, Tokyo University of Science. 2004- Guest Lecturer, Industrial Applications of Computer Science and Micro Systems, Universität Karlsruhe. 2005-2010 Assistant Professor, Department of Mechanical Engineering,

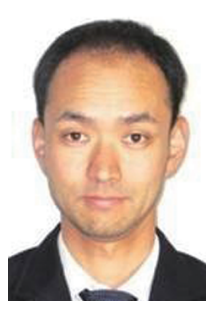
Tokyo University of Science. 2007-2010 Visiting Researcher, RIKEN (The Institute of Physical and Chemical Research). 2008-2009 Visiting Scholar, Department of Mechanical Engineering, University of Michigan. 2010- Junior Associate Professor, Department of Mechanical Engineering, Tokyo University of Science. Main Works: "Slip-adaptive Walk of Quadruped Robot," J. of Robotics and Autonomous Systems, Vol.53, No.2, pp. 124-141, 2005. Membership in Academic Societies: The Institute of Electrical and Electronics Engineers (IEEE), International Society of Biomechanics (ISB), International Society for Posture and Gait Research (ISPGR), The Japan Society of Mechanical Engineers (JSME), The Robotics Society of Japan (RSJ), Society of Biomechanisms Japan (SOBIM). 